\title{
EFEITOS DO INTERVALO PÓS-CONHECIMENTO DE RESULTADOS (CR) NA AQUISIÇÃO DE HABILIDADES MOTORAS: ESTUDO PILOTO
}

\author{
Márcio Mário Vieira \\ Faculdade Estácio de Sá de Belo Horizonte, Belo Horizonte, Minas Gerais, Brasil \\ Herbert Ugrinowitsch \\ Universidade Federal de Minas Gerais, Belo Horizonte, Minas Gerais, Brasil \\ Rodolfo Novellino Benda \\ Universidade Federal de Minas Gerais, Belo Horizonte, Minas Gerais, Brasil
}

\begin{abstract}
Resumo
$\mathrm{O}$ momento em que se fornece conhecimento de resultados (CR) envolve três intervalos de tempo, sendo um deles o pós-CR, intervalo entre a apresentação do $\mathrm{CR}$ e a próxima tentativa. Este estudo investigou a influência deste intervalo na aquisição de habilidades motoras. Utilizou-se um aparelho composto por seis recipientes em que se realizou transporte de bolas manipulando-se a sequência e tempo alvo. $\mathrm{O}$ estudo foi constituído de fase de aquisição e testes com três grupos: sem intervalo pós-CR e com intervalos de 5 e 10 segundos. O teste de Kruskal-Wallis não registrou diferenças significantes $(p>0,05)$ entre grupos no teste. Concluiu-se que o intervalo pós-CR não influenciou a aprendizagem motora, o que pode ser explicado pela ausência do intervalo pré-CR, o que sugere certa relação entre esses intervalos no processo de aquisição de habilidades motoras.
\end{abstract}

Palavras-chave: Habilidade Motora. Retroalimentação. Aprendizagem.

\section{Introdução}

A aprendizagem resulta de mudanças nos domínios cognitivo, gem motora, essas alterações levam a um aumento de competência que pode ser inferido pelo desempenho e que deve ser duradouro (GODINHO; MENDES, 1996). Para que essas mudanças possam ocorrer, alguns fatores como o feedback e prática são importantes para o processo de aquisição de habilidades motoras (BILODEAU; BILODEAU, 1958; GODINHO; MENDES, 1996). Esta importância do feedback já havia sido destacada por Trowbrigde e Cason (1932), quando sugeriram que a prática sem o conhecimento da aproximação da ação à meta real não diminui o erro inicial. Assim, o feedback é considerado como toda informação sobre o movimento realizado, 
sendo dividido em feedback intrínseco (informação oriunda do próprio indivíduo) e feedback extrínseco (informação vinda de fontes externas ao indivíduo) (SALMONI, SCHMIDT, WALTER, 1984).

O conhecimento de resultado (CR), uma subcategoria do feedback extrínseco, consiste na informação fornecida ao executante sobre o resultado de sua ação em relação ao objetivo da tarefa (MAGILL, 1994; SCHMIDT; LEE, 1999), e a manipulação dessa variável quanto ao aspecto temporal resulta em três intervalos: pré-CR, intervalo entre o término de uma tentativa e a apresentação do CR (SWINNEN et al, 1990); pós-CR, intervalo de tempo entre a apresentação do CR e o início da próxima tentativa; e o intervalo intertentativas, o intervalo de tempo entre duas tentativas (SCHMIDT; LEE, 1999).

A dificuldade para se isolar o intervalo pós-CR e também os demais intervalos de tempo do CR tem produzido evidências contraditórias quanto a sua função na aquisição de habilidades motoras (SALMONI, SCHMIDT, WALTER, 1984; TRAVLOS; PRATT, 1995; TRAVLOS, 1999). No intervalo pós-CR, o feedback intrínseco é comparado ao $\mathrm{CR}$ e a tentativa seguinte é planejada, momento que ocorre a comparação entre a referência existente na memória e o resultado obtido (TRAVLOS; PRATT, 1995; TRAVLOS, 1999).

Ao manter constante o intervalo pré-CR e manipulando-se o intervalo pós-CR, ou o oposto, altera-se o intervalo intertentativas, o que resulta em duas variáveis independentes (SALMONI, SCHMIDT, WALTER, 1984). Por exemplo, quando os intervalos de pré ou pósCR semelhantes foram comparados ao intervalo intertentativas, tanto os grupos de intervalo pré-CR como de pós-CR registraram melhores resultados (GALLAGHER; THOMAS, 1980). Contudo, resultados contraditórios foram observados nos grupos com intervalos pré-CR e pós-CR diferentes quando comparados com o intervalo intertentativas, pois apenas o intervalo pós-CR apresentou desempenho superior (SIMMONS; SNYDER, 1983). Outros estudos indicaram melhores resultados dos grupos de intervalo pós-CR quando comparados aos grupos de intervalo pré-CR (WEINBERG; GUY; TUPPER, 1964; RAMELLA, 1983).

A extensão dos intervalos de tempo de pós-CR estudados variaram de 1 segundo (WEINBERG; GUY; TUPPER, 1964) até 1 hora (BILODEAU; BILODEAU, 1958). Dentre esses, os estudos que utilizaram intervalos entre 5 e 10 segundos apresentaram melhores resultados (RAMELLA, 1983; MAGILL, 1988; GODINHO; MEN- 
DES, 1996), o que não é observado nos intervalos fora dessa faixa de tempo (TRAVLOS; PRATT, 1995; TRAVLOS, 1999). Esse resultado não foi verificado no estudo de Vieira et al. (2006) que investigaram o efeito do intervalo pós-CR na aquisição de uma tarefa de arremesso. Não foi encontrada diferença significante entre os grupos sem pós$\mathrm{CR}$, cinco segundos e dez segundos de intervalo pós-CR na aquisição do arremesso da bocha. Contudo, nessa tarefa havia um intervalo complementar entre o término do lançamento e o momento que a bola para que pode ter proporcionado igualdade entre os grupos, e assim interferindo no papel do pós-CR.

Em sua maioria os estudos sobre o intervalo pós-CR utilizaram tarefas simples como habilidades gráficas (desenho de linhas) (MCGUIGAN; CROCKETT; BOLTON, 1960), apertar botões (MAGILL, 1988) ou mover uma alavanca (MAGILL, 1977; RAMELLA, 1983; SCHMIDT; SHEA, 1966; SIMMONS; SNYDER, 1983; WEINBERG; GUY; TUPPER, 1964) e não encontraram resultados claramente favoráveis ao efeito do pós $\mathrm{CR}$ na aquisição de habilidades motoras. Dessa forma, com o baixo número de estudos sobre o intervalo pós-CR que apresentaram resultados favoráveis (GALLAGHER; THOMAS, 1980; MAGILL, 1988; RAMELLA, 1983; WEINBERG; GUY; TUPPER, 1964), e a inconsistência nos resultados dos demais trabalhos (MAGILL, 1977; MAGILL, 1988; MCGUIGAN; CROCKETT; BOLTON, 1960; SCHMIDT; SHEA, 1966; SIMMONS; SNYDER, 1983; VIEIRA et al., 2006) requer-se maior compreensão dessa variável na aprendizagem motora. Assim, o presente estudo teve como objetivo verificar a influência do intervalo pós-CR na aquisição de uma habilidade motora de posicionamento manual.

\section{Método}

Amostra

Participaram do estudo 15 universitários voluntários de ambos os sexos, com faixa etária entre 18 e 35 anos (media $=23,19+3,18$ ), novatos na tarefa e com consentimento livre e esclarecido. O estudo foi aprovado pelo Comitê de Ética em Pesquisa da Universidade Federal de Minas Gerais, sob parecer de número ETIC 297/05. 
Instrumento e Tarefa

Foi utilizado um aparelho composto de uma plataforma contendo seis recipientes enumerados de um a seis, uma central de controle ligada a um microcomputador, constituída por diodos que fornecem estímulo visual para iniciar a tarefa e uma chave de resposta para controle das medidas de tempo de reação e tempo de movimento. Um software foi desenvolvido para medida e armazenamento dos tempos fornecidos pelo aparelho (Figura 1). A tarefa constituiu no transporte de bolas de tênis (transporte manual) em sequenciamento e tempo alvo pré-determinados.

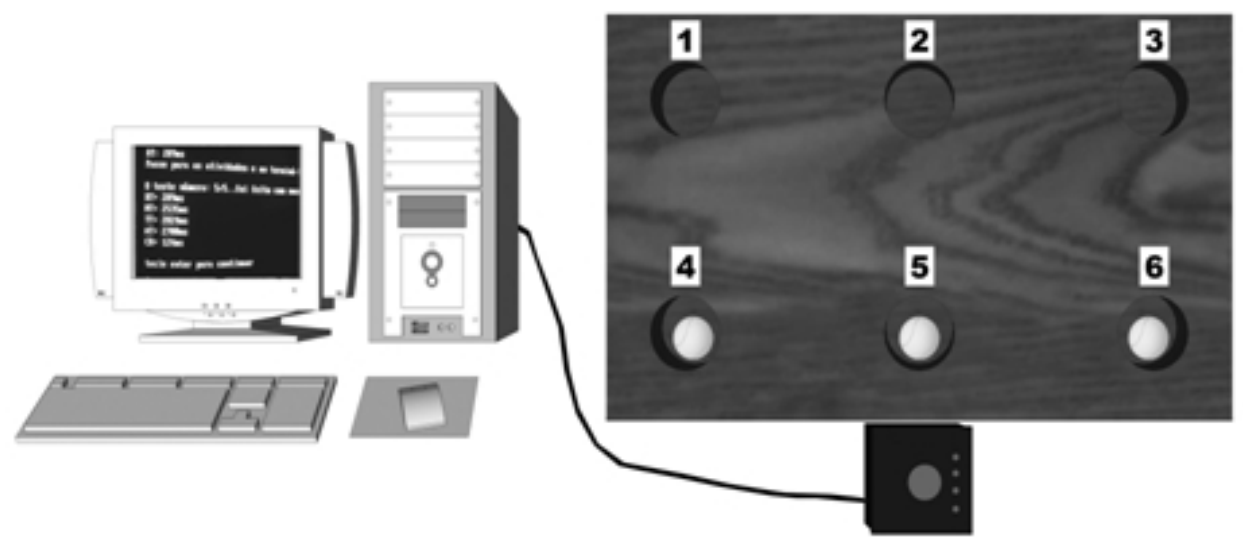

Figura 1: Aparelho utilizado no experimento

Delineamento e procedimentos experimentais

Os sujeitos foram divididos aleatoriamente em três grupos experimentais $(\mathrm{n}=5)$ : sem intervalo pós-CR ( $\mathrm{s} /$ pós-CR), com intervalo de 5 (5s) e 10 segundos (10s). Para a fase de aquisição, utilizou-se um sequenciamento 4.2 / 5.3 / 6.1 (transportar a bola do recipiente quatro para o recipiente dois, do recipiente cinco para o recipiente três e do recipiente seis para o recipiente um), em um tempo alvo de $2700 \mathrm{mi}-$ lissegundos. Para os testes utilizou-se o sequenciamento 6.1/5.3/4.2 e um novo tempo alvo de 2300 milissegundos. A fase de aquisição foi encerrada quando os sujeitos atingiram o desempenho de duas tentativas consecutivas dentro de uma faixa de erro absoluto de 40 milissegundos acima ou abaixo do valor da meta estipulada. Esse critério foi adotado para garantir que os grupos apresentassem desempenhos se- 
melhantes ao fim da fase de aquisição, e assim se pudesse avaliar o efeito da variável independente. Posteriormente, foi realizado o teste de transferência com dez tentativas sem CR quatro minutos após o término da fase de aquisição e a retenção da transferência dez minutos após o primeiro teste. Esses tempos para aplicação dos testes foram determinados por estudo piloto mostrando-se suficientes para identificar os efeitos duradouros característicos da aprendizagem. O CR fornecido foi verbal em magnitude e direção.

\section{Análise Estatística}

Inicialmente foi analisada a normalidade da distribuição dos dados por meio do teste de Shapiro-Willks. Como esse pressuposto não foi observado optou-se por utilizar a análise estatística não paramétrica. Assim, para analisar possíveis diferenças entre o desempenho dos grupos utilizou-se o teste de Kruskall-Wallis.

\section{Resultados}

Os dados da fase de aquisição e testes foram distribuídos em blocos de cinco tentativas. Na fase de aquisição foram registrados o primeiro e último blocos de tentativas, pois em virtude do desempenho critério os sujeitos tiveram diferentes quantidades de prática.

Erro Absoluto

O teste de Kruskal-Wallis não encontrou diferenças significantes intergrupos (Figura 2) no último bloco da fase de aquisição $[\mathrm{H}(2, \mathrm{n}=15)=4.88 \mathrm{p}=.087]$; no primeiro bloco do teste de transferência $1[\mathrm{H}(2, \mathrm{n}=15)=.38 \mathrm{p}=.82]$; no segundo bloco do teste de transferência $1[\mathrm{H}(2, \mathrm{n}=15)=1.82 \mathrm{p}=.4]$; no primeiro bloco do teste de transferência $2[\mathrm{H}(2, \mathrm{n}=15)=2.34 \mathrm{p}=.31]$ e no segundo bloco do teste de transferência $2[\mathrm{H}(2, \mathrm{n}=15)=1.68 \mathrm{p}=.43]$. 


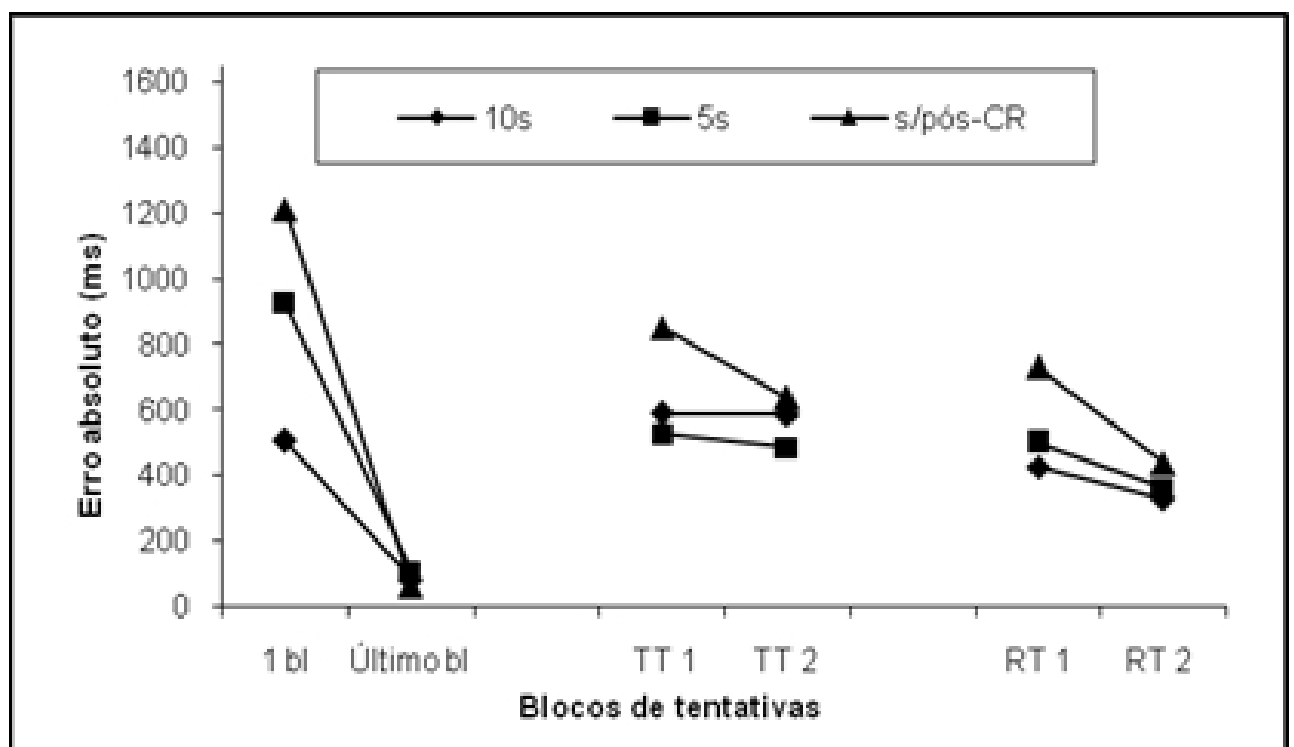

Figura 2: Média do Erro Absoluto em blocos de 5 tentativas na fase de aquisição (primeiro e último bloco) e testes de transferência.

\section{Desvio Padrão do Erro Absoluto}

O teste de Kruskal-Wallis não encontrou diferenças significantes intergrupos (Figura 3) no último bloco da fase de aquisição $[\mathrm{H}(2, \mathrm{n}=15)=1.22 \mathrm{p}=.54]$; no primeiro bloco do teste de transferência $1[\mathrm{H}(2, \mathrm{n}=15)=.38 \mathrm{p}=.83]$; no segundo bloco do teste de transferência $1[\mathrm{H}(2, \mathrm{n}=15)=.98 \mathrm{p}=.61]$; no primeiro bloco do teste de transferência $2[\mathrm{H}(2, \mathrm{n}=15)=.18 \mathrm{p}=.91]$ e no segundo bloco do teste de transferência $2[\mathrm{H}(2, \mathrm{n}=15)=2.34 \mathrm{p}=.31]$.

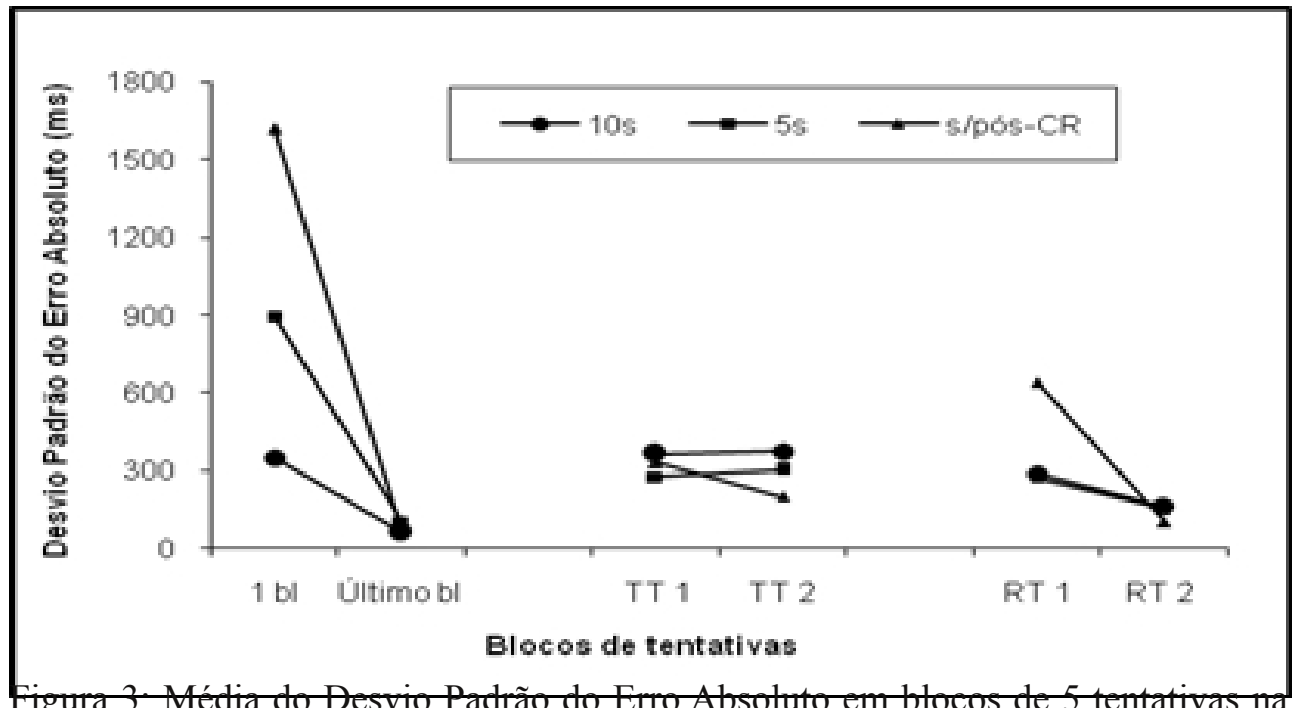

Figura 3. Média do Desvio Padrão do Emo Absotuto em blocus de
fase de aquisição (primeiro e último bloco) e testes de transferência.

Pensar a Prática, Goiânia, v. 16, n. 3, p. 619-955, jul./set. 2013 
Devido à utilização de desempenho critério, foi analisado também o número de tentativas necessárias para atingi-lo, e os resultados mostraram que o grupo 10s necessitou de mais tentativas que o grupo 5 s e sem pós-CR (Figura 4). Entretanto, o teste de Kruskal-Wallis não encontrou diferença significativa $[\mathrm{H}(2, \mathrm{n}=15)=2.07 \mathrm{p}=.36]$.

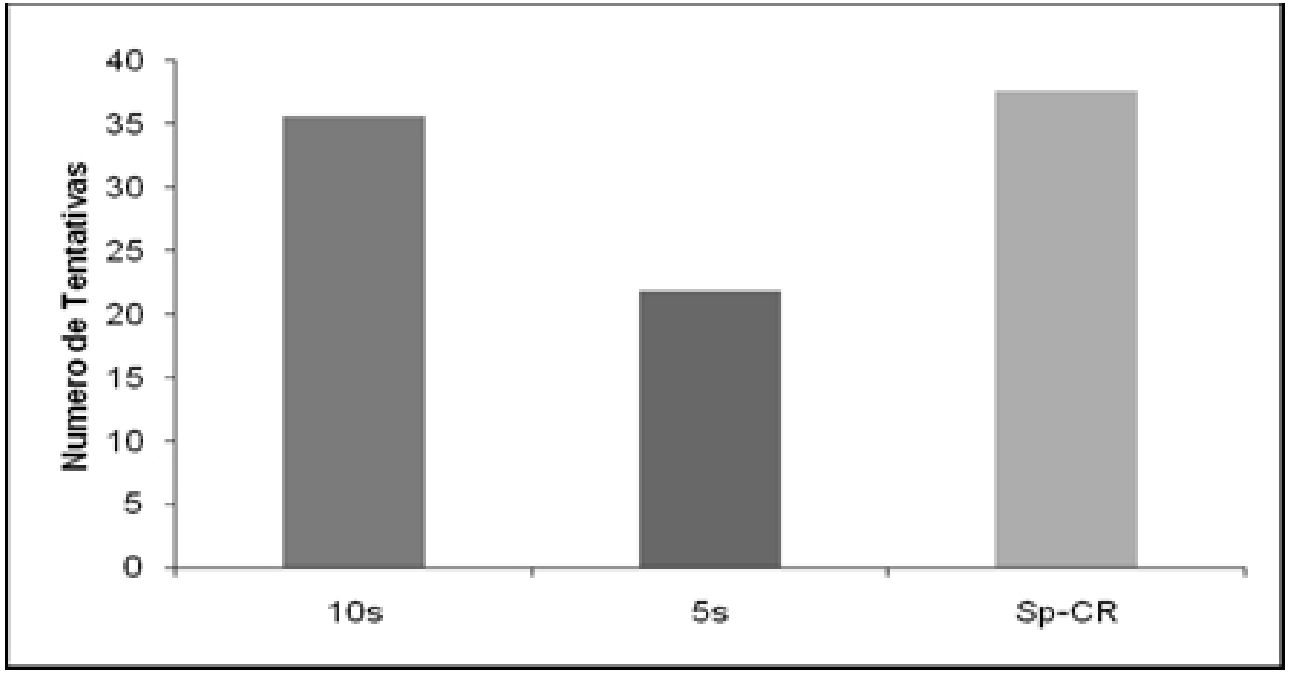

Figura 4: Média das tentativas por grupo na fase de aquisição.

\section{Discussão}

As análises das medidas de desempenho (EA e seu desvio padrão) não encontraram diferenças entre os grupos, sugerindo que o intervalo pós-CR não representou uma variável capaz de influenciar a aprendizagem da habilidade de transporte manual. Esses achados não corroboram com os estudos de Ramella (1983), Weinberg, Guy e Tupper (1964) e Gallagher e Thomas (1980), nos quais as faixas de intervalos pós-CR apresentaram melhores resultados que grupos sem o intervalo pós-CR ou com intervalos de tempo superiores a 12 segundos.

As declarações de Godinho e Mendes (1996) e os achados dos estudos de Simmons e Snyder (1983) e Vieira et al. (2006) corroboram os resultados do presente estudo, no qual o intervalo pós-CR não influenciou a aquisição de habilidades motoras. Assim, no presente estudo alguns aspectos podem ter interferido de forma que o efeito do intervalo pós-CR não tenha sido evidenciado.

$\mathrm{O}$ primeiro aspecto se relaciona a uma demora no processo de comparação e elaboração da resposta. A ausência do intervalo pós-CR poderia não proporcionar tempo suficiente para as operações corres- 
pondentes ao intervalo pós-CR, ou ao contrário, intervalos longos poderiam interferir na capacidade de atenção influenciando a memória. Esse aspecto levaria os indivíduos a realizarem a comparação e preparação da estratégia com tentativas de atraso (VIEIRA et al., 2006). Uma tendência a ser levada em consideração seria análise do número de tentativas da fase de aquisição, na qual os grupos sem pós-CR e 10 s necessitaram de maiores quantidades de prática em relação ao grupo $5 \mathrm{~s}$ que necessitou de menor quantidade de prática, mesmo a análise estatística não registrando diferença significativa.

Outra explicação refere-se às operações que provavelmente ocorrem durante o intervalo pós-CR, comparação entre o feedback intrínseco e o CR, bem como a elaboração da estratégia para a execução da tentativa seguinte (SCHMIDT, 1988). Assim, há aparentemente uma relação entre o intervalo pré-CR, no qual o feedback intrínseco é processado, e a efetividade do intervalo pós-CR. Essa proposição tem suporte nos resultados favoráveis dos grupos com intervalo pós-CR dos estudos de Gallagher e Thomas (1980), Magill (1988), Ramella (1983) e Weinberg, Guy e Tupper (1964) que apresentaram intervalos pré-CR fixos em seus delineamentos. Por sua vez, o presente estudo não utilizou intervalo de atraso pré-CR, o que talvez não tenha favorecido a utilização do intervalo pós-CR. Contudo, essas considerações são limitadas devido ao baixo número de indivíduos utilizado no estudo.

Em suma, o intervalo pós-CR, na ausência de intervalo pré-CR, não mostrou qualquer efeito na aquisição de uma habilidade motora de transporte manual. Sugere-se que novos estudos sejam conduzidos de modo a testar a hipótese de que o efeito do intervalo pós-CR é mais bem observado quando há também um intervalo pré-CR. Assumindose como requisito principal uma amostra de tamanho adequado as características do estudo.

Effects of post-knowledge of results (KR) interval in motor skill acquisition: pilot study

\begin{abstract}
Temporal locus of knowledge of results (KR) involves three time intervals. Among them, post-KR interval is the time between KR presentation and the next trial. This study investigated the effect of post-KR interval in motor skill acquisition. It was used a device consisting of six containers where balls should be transported. It was manipulated the sequence and target time. The study presented acquisition phase and tests with three groups: no post-KR interval and intervals of 5 and 10 seconds
\end{abstract}


of post-KR. Kruskal-Wallis test did not register significant differences $(p>0.05)$ among groups in testes. It was concluded that post-KR interval did not influence motor learning, what could be explained for pre-KR interval absence, suggesting a certain relation between intervals in motor skills acquisition process.

Keywords: Motor Skill. Feedback. Learning.

Efectos del intervalo pós-conocimiento de los resultados (CR) en la adquisición de las habilidades motoras: estudio piloto

\section{Resumen}

El momento en el que aporta el conocimiento de los resultados (CR) implica tres intervalos de tiempo, siendo uno de ellos el post-CR, intervalo de tiempo entre la presentación de los CR y el siguiente intento. Este estudio investigó la influencia de este intervalo en la adquisición de las habilidades motoras. Utilizamos una herramienta formada por seis contenedores en los que se celebraron las pelotas por transporte de manipular la secuencia y tiempo meta. El estudio se compone de fase de adquisición y exámenes con tres grupos: sin intervalos post-CR y con intervalos de 5 y 10 segundos. La prueba de Kruskal-Wallis no se observaron diferencias significativas $(\mathrm{p}>0,05)$ entre los dos grupos en la prueba. Se llegó a la conclusión que el intervalo después de CR no influyó en el aprendizaje motor, que puede explicarse por la ausencia de rango pre-CR, que denota una cierta relación entre estos intervalos en el proceso de adquisición de las habilidades motoras.

Palabras clave: Destreza Motora. Retroalimentación. Aprendizaje.

\section{Referências}

BILODEAU, E. A.; BILODEAU, I. M. Variable frequency knowledge of results and the learning of a sample skill. Journal of Experimental Psychology, v. 55, p. 379-383, 1958.

GALLAGHER, J. D.; THOMAS, J. R. Effects of varying post-KR intervals upon children's motor performance. Journal of Motor Behavior, v. 12, n. 1, p. 41-56, mar., 1980.

GODINHO, M.; MENDES, R. Aprendizagem Motora: informação de retorno sobre o resultado. Cruz Quebrada: FMH, 1996.

MAGILL, R. A. The processing of knowledge of results information for a serial-motor task. Journal of Motor Behavior, v. 9, p. 113-118, 1977.

. Activity during the post-knowledge of result interval can benefit motor skill learning. In: MEIJER, O. G., ROTH, K. (Eds.) Com- 
plex Movement Behavior: The motor-action controversy. Amsterdam: Elsevier, 1988. p. 231-246.

. The influence of augmented feedback on skill learning depends on characteristics of the skill and the learner. Quest, v. 46, n. 3, p.314-327, 1994.

MCGUIGAN, F J.; CROCKETT, F.; BOLTON, C. The effect of knowledge of results before and after a response. The Journal of General Psychology, v.63, n. 1, p. 51-55, 1960.

RAMELLA, R. J. Processing, knowledge of results, and a multi-dimensional task. Perceptual and Motor Skills, v. 57, n. 1, p. 43-48, 1983.

SALMONI, A. W.; SCHMIDT, R. A.; WALTER, C. B. Knowledge of results and motor learning: a review and critical reappraisal. Psychological Bulletin, v. 95, n. 3, p.355-386, maio, 1984.

SCHMIDT, R. A. Motor control and learning: a behavioral emphasys. 2. ed. Champaign: Human Kinetics, 1988.

SCHMIDT, R. A.; LEE, T. D. Motor control and learning: a behavioral emphasis. 3. ed. Champaign: Human Kinetics, 1999.

SCHMIDT, R. A.; SHEA, J. B. A note on delay of knowledge of results in positioning responses. Journal of Motor Behavior, v. 8, p. 129-131, 1976.

SIMMONS, R. W.; SNYDER, R. J. Variation of temporal locus of knowledge of results: effects on motor performance of a simple task. Perceptual and Motor Skills, Missoula, v. 56, p. 399-404, 1983.

SWINNEN, S. P. et al. Information feedback for skill acquisition: Instantaneous knowledge results degrade learning. Journal of Experimental Psychology, v. 16, n. 4, p. 706-716, 1990.

TRAVLOS, A. K. Re-examining the temporal locus of knowledge of results (KR): A self-paced approach to learning. Perceptual and Motor Skills, v.89, p.1073-1087, 1999. 
TRAVLOS, A. K.; PRATT, J. The temporal locus of knowledge of results: a meta-analytic review. Perceptual and Motor Skills, Missoula, v. 80, n. 1, p. 3-14, fev., 1995.

TROWBRIDGE, M. H.; CASON, H. An experimental study of Thorndike's theory of learning. Journal of General Psychology, Washington, v. 7, p. 245-260, 1932.

VIEIRA, M. M. et al. Os efeitos do intervalo pós-conhecimento de resultados na aquisição do arremesso da bocha. Revista Portuguesa de Ciências do Desporto, Porto, v.6, n.1. p.50-54, jan., 2006.

WEINBERG, D. R.; GUY, D. E.; TUPPER, R. W. Variations of post feedback interval in simple motor learning. Journal of Experimental Psychology, v.67, p.98-99, 1964.

Recebido em: 06/04/2012

Revisado em: 12/09/2012

Aprovado em: 24/01/2013

Endereço para correspondência

rodolfobenda@yahoo.com.br

Rodolfo Novellino Benda

Universidade Federal de Minas Gerais

Escola de Educação Física, Departamento de Educação Física

Av. Presidente Carlos Luz, 4664

Pampulha

31310-250 - Belo Horizonte, MG - Brasil 